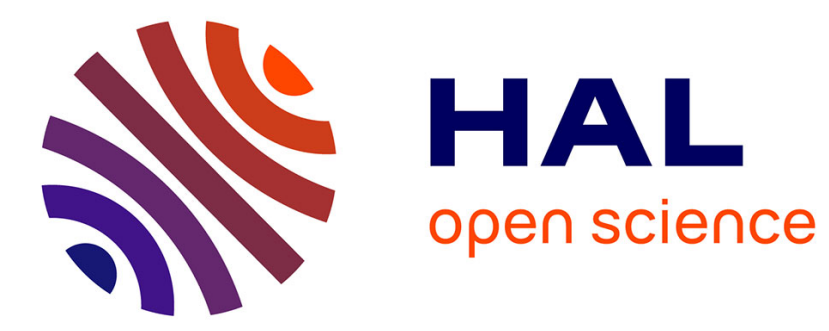

\title{
Dire le paysage-patrimoine dans la Convention européenne du paysage
}

Mariagrazia Margarito

\section{To cite this version:}

Mariagrazia Margarito. Dire le paysage-patrimoine dans la Convention européenne du paysage. Culture et Musées, 2019, 33, pp.157-179. 10.4000/culturemusees.3210 . hal-03254257

\section{HAL Id: hal-03254257 \\ https://hal.science/hal-03254257}

Submitted on 8 Jun 2021

HAL is a multi-disciplinary open access archive for the deposit and dissemination of scientific research documents, whether they are published or not. The documents may come from teaching and research institutions in France or abroad, or from public or private research centers.
L'archive ouverte pluridisciplinaire HAL, est destinée au dépôt et à la diffusion de documents scientifiques de niveau recherche, publiés ou non, émanant des établissements d'enseignement et de recherche français ou étrangers, des laboratoires publics ou privés. 


\section{OpenEdition}

Journals

\section{Culture \& Musées}

Muséologie et recherches sur la culture

$33 \mid 2019$

La fabrique des patrimoines européens au XXIe siècle

Dossier

\section{Dire le paysage-patrimoine dans la Convention européenne du paysage}

Landscape and Heritage in the Words of the European Landscape Convention

Decir el patrimonio-paisaje en el Convenio europeo del paisaje

MARIAGRAZIA MARGARITO

p. $157-179$

https://doi.org/10.4000/culturemusees.3210

\section{Résumés}

Français English Español

Cet article sur la Convention européenne du paysage ou CEP (Florence 2000) cherche à approfondir certaines réflexions concernant le rapport entre paysage et patrimoine, notamment du point de vue du patrimoine européen. Dans le cadre des sciences du langage et de l'analyse du discours de l'école française sont étudiés des items lexicaux et phrastiques du texte de la CEP ainsi que des notes d'analyse du "Glossaire » (versions 2012 et 2013) rédigé par un groupe de travail du Conseil de l'Europe. Les définitions du paysage et du patrimoine sont comparées avec d'autres traités internationaux, dont le plus célèbre : la Convention concernant la protection du patrimoine mondial de l'Unesco (1972). Par ailleurs, nous tentons de mettre en évidence des réflexions épistémiques interrogeant la notion de paysage comme " élément important de la qualité de vie des populations dans les milieux urbains et dans les campagnes, dans les territoires dégradés comme dans ceux de grande qualité, dans les espaces remarquables comme dans ceux du quotidien » (Préambule de la CEP), mais aussi le paysage comme composante du patrimoine et de l'identité européenne, la « perception » individuelle et sociale du paysage suite aux affirmations de la CEP.

Looking at the European Landscape Convention (ELC, Florence 2000), this paper aims to highlight certain aspects of the relationship between landscape and European cultural heritage. Drawing on linguistics and discourse analysis, the paper provides an analysis of the ELC text's lexical and phrasal items, as well as of the 2012-2013 "Glossary" from the Council of Europe. The definitions of landscape and heritage are compared to those of other international conventions such as the 1972 Unesco "Convention Concerning the Protection of the World Cultural and Natural Heritage". The paper highlights research findings pointing to the landscape as "an important part of the quality of life for people everywhere: in urban areas and in the countryside, in degraded areas as well as in areas of high quality, in areas recognised as being of outstanding 
beauty as well as everyday areas" (ELC Preamble), landscape as a part of European heritage and identity, as well as the individual and social "perception" of the landscape as stated in the ELC.

Este estudio sobre el Convenio Europeo del Paisaje (Florencia 2000) (CEP) busca poner en relieve algunos de los datos relativos a la relación entre paisaje y patrimonio, en particular el patrimonio europeo. Conforme con las disciplinas lingüísticas y del análisis del discurso de la escuela francesa, se presentan comentarios sobre los ítems lexicales y enunciados frásticos del CEP y notas de análisis sobre el «Glosario» (versiones 2012-2013) redactado por un Grupo de trabajo del Consejo de Europa. Las definiciones de paisaje y patrimonio son comparadas con las de otros tratados internacionales, incluyendo la más conocida «Convención de la Unesco para la Protección del Patrimonio Mundial» (1972). También evidenciamos las reflexiones sobre el paisaje como «un elemento importante de la calidad de vida de las personas: en las zonas urbanas y en el campo, en territorios degradados y aquellos de alta calidad, en espacios remarcables como en aquellos de la vida cotidiana» (Preámbulo del CEP), sobre el paisaje como componente del patrimonio y de la identidad europea, sobre la «percepción» individual y social del paisaje según las declaraciones del CEP.

\section{Entrées d'index}

Mots-clés : convention européenne du paysage, patrimoine, paysage, analyse du discours, langue française

Keywords: European Landscape Convention, heritage, landscape, discourse analysis, French language

Palabras clave: Convenio europeo del paisaje, patrimonio, paisaje, análisis de discurso, lengua francesa

\section{Notes de la rédaction}

Manuscrit reçu le 21 novembre 2018

Version révisée reçue le 2 octobre 2019

Article accepté pour publication le 10 octobre 2019

\section{Texte intégral}

Ouverte à Florence le 20 octobre 2000 à la signature des États membres du Conseil de l'Europe, entrée en vigueur le 1er mars 2004, regroupant 38 États parties et deux États signataires, la Convention européenne du paysage (CEP) est peut-être moins connue du grand public que d'autres conventions et traités internationaux. Appelée aussi Convention de Florence, elle se présente comme " premier traité international consacré à l'ensemble des dimensions du paysage européen ». Elle se compose d'un préambule et de 18 articles regroupés en quatre chapitres ${ }^{1}$. Ses objectifs principaux sont de « promouvoir la protection, la gestion et l'aménagement des paysages », et « d'organiser la coopération internationale dans ce domaine ».

2 N'étant pas une loi, comme toutes les conventions et les traités internationaux, la CEP est un document prescriptif, quoique à faible valeur perlocutoire, et institutionnel, qui vise un consensus général, cherche à anticiper et à diffuser des opinions, des valeurs, comme en témoignent les formules axiologiques que l'on retrouve dans la langue de rédaction : " développement durable ", " équilibre harmonieux ", " ressources communes ", " sensibilisation à la valeur des paysages », etc.. Il vise également à faire évoluer voire à former les mentalités, à mettre en place des activités prospectives « pour la mise en valeur, la restauration ou la création de paysages » (CEP, chap. I, art. 1) à partir d'un des pivots de cette convention, l'assertion préliminaire que " le paysage constitue un élément essentiel du bien-être individuel et social » (préambule de la CEP).

3 Nous proposons ici d'analyser les éléments de la CEP qui concernent le paysage et le patrimoine, et de rendre compte de travaux et de documents qui accompagnent et soutiennent cette convention. Le suivi de son actualisation a en outre fait surgir la nécessité de définitions précises sur les mots «pour la dire $»^{2}$. Les discours, la part langagière des traités nationaux ou internationaux, sont indispensables à la vie des institutions qui les produisent. Soumis à des règles de normalisation, les luttes et débats qui sont à l'origine de ces textes sont estompés par la généricité et l'ambiguïté du 
rendu textuel où la pacification des contraires est obligatoire, afin que l'institution continue à transmettre son image de marque à valeur d'autorité universelle. En nous penchant sur ces textes, nous allons examiner comment paysage et patrimoine acquièrent existence dans et par le discours. Nous nous trouverons alors face à un dispositif langagier communicationnel bien réglementé et ritualisé : aucune individualisation des auteurs (c'est l'institution qui parle), pas d'effets de style. Au niveau lexical, chaque mot a été pesé et résulte presque toujours d'un compromis accepté par une assemblée de membres dont au départ les opinions et les engagements politiques pouvaient être très différents. Le résultat est un agencement discursif lisse, sans pics rhétoriques ni pathos. Les données lexicales, les items discursifs qui dans ce texte sont une sorte de dénominateur commun de consensus entre les parties qui adhèrent à la Convention, restent souvent vagues, ambigus. Dans l'optique linguistique qui est la nôtre, ce flou, ces ambiguités dont toute langue est pourvue, peuvent être envisagés comme une richesse, l'ouverture de plusieurs possibles où locuteurs et destinataires de messages peuvent façonner la réalité des discours.

\section{Corpus de travail et corpus d'appui}

Pour cette recherche, que nous situons dans le cadre des disciplines linguistiques et de l'analyse du discours, notre corpus de travail est la version française de la CEP3, à laquelle nous ajoutons la Recommandation $\mathrm{CM} / \operatorname{Rec}(2008) 3$ du Comité des ministres du Conseil de l'Europe aux États membres sur les orientations pour la mise en ouvre de la Convention européenne du paysage, adoptée le 6 février 20084. S’y ajoutent des documents relatifs au " Projet "Un glossaire de la Convention européenne du paysage pour L6” »5. Nous allons nous servir, en outre, d'un corpus d'appui constitué par les Actes annuels des réunions des ateliers pour la mise en œuvre de la Convention européenne du paysage, à partir de la première réunion qui a eu lieu à Strasbourg en $2002^{6}$. Ces ateliers thématiques nourrissent pratiques et projets pour l'application de la CEP aux niveaux local, national et international, ils sont des creusets de réflexions, des émanations des forums de propositions et de débats sur de nouveaux concepts7. Chaque intervention est signée.

5 En résumant, nous avons affaire à ce qu'on peut appeler un " discours instituant » (Oger \& Ollivier-Yanniv, 2003 : s. p.), c'est-à-dire la CEP dans son aspect normatif, énoncé officiel où les énonciateurs sont gommés, subsumés par l'organisme international, et des discours, institutionnels eux aussi, produits par des commissions, des groupes de travail qui soutiennent, corroborent, prolongent par des documents les projets d'actualisation, de révision ou d'explicitation des données du discours instituant, qu'ils supportent et sur lequel ils s'appuient. Dans son déroulement discursif, la CEP suit des routines linguistiques typiques de la standardisation des discours institutionnels (Krieg-Planque, 2012 : 106 sqq.) : découpage textuel, organisation en paragraphes, séquences de participes présents, d'adjectifs, de participes passés à valeurs adjectivale : " considérant que, soucieux de, notant que, persuadés que, etc. " (cf. notamment le préambule de la $\mathrm{CEP}^{8}$ ). Malgré le lissage communicationnel du discours constituant, c'est-à-dire « le gommage des formes de diversité et d'hétérogénéité en général » (Oger \& Ollivier-Yanniv, 2006 : s. p.), d’où il résulte, par exemple, une atténuation maximale d'éventuelles données conflictuelles ou pouvant engendrer des interprétations adverses, le texte de la CEP présente des items lexicaux et phrastiques aptes à susciter intérêt et questionnements.

$6 \quad$ Dans une étude précédente (Margarito, 2011 : 283 sqq.), nous nous étions penchée sur les choix lexicaux de la CEP : au-delà du lissage des données discursives, que nous venons d'évoquer, ils révèlent des visées nouvelles, inattendues parfois dans les textes des institutions internationales, et même une forte valeur morale attribuée à " paysage ». Nous avions relevé au niveau lexical et phrastique des évaluatifs porteurs d'affectivité, peu fréquents dans les textes des grandes institutions. On trouve en effet " épanouissement des êtres humains ", " bien-être individuel et social », et presque le devoir de « jouir des paysages ». Dans un atelier, l'argumentation de Felice Spingola 
pour qui la «perception du paysage » non seulement individuelle, mais sociale va bien au-delà des paysages d'excellence, des territoires exceptionnels, étaye notre affirmation. Nous reviendrons sur la notion de perception, qui « inscrit le paysage parmi les droits de l'homme : le droit au paysage n'est pas une prérogative de peu, mais de tous » (Spingola, A4, 2007 : 243).

\section{Quelques points saillants de la Convention européenne du paysage}

7 Nous allons aborder ce qui nous semble être quelques points forts de cette convention, compte tenu de la « fabrique » des patrimoines européens au courant de notre siècle par les discours des grandes institutions internationales.

8 En schématisant, d'après le préambule et l'article 1 de la CEP, nous voyons que pour la CEP le paysage est :

- la « partie de territoire telle que perçue par les populations, dont le caractère résulte de facteurs naturels et/ou humains et de leurs interrelations »;

- un « élément important de la qualité de vie des populations »;

- un « élément essentiel du bien-être individuel et social »; et qu'il « concourt à l'élaboration des cultures locales et [...] représente une composante fondamentale du patrimoine culturel et naturel de l'Europe, contribuant à l'épanouissement des êtres humains et à la consolidation de l'identité européenne ».

L'article 2 précise le champ d'application de la Convention : elle convient à « tout le territoire des Parties et porte sur les espaces naturels, ruraux, urbains et périurbains. Elle inclut les espaces terrestres, les eaux intérieures et maritimes ». Nous soulignons la suite : " Elle concerne, tant les paysages pouvant être considérés comme remarquables, que les paysages du quotidien et les paysages dégradés. »

Cette dernière proposition doit être comparée avec la convention internationale la plus connue, centrée sur le patrimoine mondial : la Convention concernant la protection du patrimoine mondial culturel et naturel, adoptée par la Conférence générale de l'Organisation des Nations unies pour l'éducation, la science et la culture, à sa dix-septième session, à Paris, le 16 novembre $1972^{9}$, et qui se réfère au « Patrimoine mondial de l'Unesco » tel que génériquement appelé.

11 En ciblant ce qui nous occupe dans ces pages, nous comparons, en les résumant, les différents points de vue sur le patrimoine et le paysage de la Convention de l'Unesco (partie introductive) et de la CEP :

\begin{tabular}{|c|c|}
\hline $\begin{array}{l}\text { Convention de l'Unesco } \\
\text { concernant la protection du } \\
\text { patrimoine mondial culturel et } \\
\text { naturel }\end{array}$ & Convention européenne du paysage \\
\hline $\begin{array}{l}\text { Le paysage est un patrimoine. } \\
\text { Sont spécialement ciblés « certains } \\
\text { biens du patrimoine culturel et } \\
\text { naturel [qui] présentent un intérêt } \\
\text { exceptionnel qui nécessite leur } \\
\text { préservation en tant qu'éléments du } \\
\text { patrimoine mondial de l'humanité } \\
\text { tout entière ». } \\
\text { Conséquence : « il incombe à la } \\
\text { collectivité internationale tout entière } \\
\text { de participer à la protection du } \\
\text { patrimoine culturel et naturel de } \\
\text { valeur universelle exceptionnelle ». }\end{array}$ & $\begin{array}{l}\text { Le paysage est un élément d'un patrimoine plus vaste. } \\
\text { Le paysage est « tout le territoire des Parties [adhérant } \\
\text { à la CEP] » à savoir « les espaces naturels, ruraux, } \\
\text { urbains et périurbains [...] les espaces terrestres, les } \\
\text { eaux intérieures et maritimes » y inclus « les paysages } \\
\text { pouvant être considérés comme remarquables [...] les } \\
\text { paysages du quotidien et les paysages dégradés ». }\end{array}$ \\
\hline
\end{tabular}

Remarquons la séquence " patrimoine culturel et naturel », où la nature apparaît en seconde place. Presque trente ans plus tard, nous retrouverons le même ordre des 
adjectifs dans la CEP, qui révèle peut-être une conception de la nature considérée comme surplombée par la culture.

L'item "valeur universelle exceptionnelle » est repris dans les articles 1 et 2 de l'Unesco, et dans l'article 11, paragraphe 2, où il est question de l'établissement d'une " liste des biens du patrimoine culturel et du patrimoine naturel », ainsi que dans l'article 19 qui concerne les modalités d'assistance internationale pouvant être prises en compte par chaque État signataire de cette convention. Notons que le paragraphe 4 de l'article 11 cité vise une liste du " patrimoine mondial en péril » où ne peuvent figurer que des biens sur lesquels pèsent de graves dangers de disparition (guerres, grands travaux publics ou privés, calamités naturelles, développement urbain et touristique trop rapide).

14 La version Unesco récente des « Orientations devant guider la mise en œuvre de la Convention du patrimoine mondial » (juillet 2017) ${ }^{10}$ donne à lire ce que signifie " valeur universelle exceptionnelle », c'est-à-dire " une importance culturelle et/ou naturelle tellement exceptionnelle qu'elle transcende les frontières nationales et qu'elle présente le même caractère inestimable pour les générations actuelles et futures de l'ensemble de l'humanité » (chap. II.A : 49). Le Comité du patrimoine mondial (institué par l'article 8 du texte de la Convention du patrimoine mondial) identifie les critères qui, après aboutissement de toutes les démarches requises de la part des États demandeurs, permettront l'inscription d'un bien sur la liste du patrimoine mondial (III.G : 154-155). De grands moyens économiques sont prévus et réglementés par l'Unesco, dont la Convention (ne l'oublions pas) est orientée sur le patrimoine.

15 La CEP, centrée sur le paysage, prévoit dans son "Suivi de la mise en œuvre de la Convention » (art. 10) un Prix du paysage (généralement bisannuel) à valeur honorifique pour une réalisation exemplaire de bonnes pratiques dans l'aménagement, la préservation, la sauvegarde de paysages intégrés dans les politiques territoriales nationales, régionales ou locales, suivant l'optique de la CEP. Le prix est décerné aux projets de sensibilisation au paysage, quel qu'il soit, et non pas à des lieux remarquables, exceptionnels, significatifs de par leur histoire ou leur mise en scène plus ou moins récente. Nous avons alors des différences évidentes sur les plans économique, social et politique : d'une part l'attribution d'un label exceptionnel (Unesco) pour des biens paysagers labellisés patrimoine mondial, mis ou remis en état grâce à d'imposants soutiens économiques, et à l'engagement de la part des États destinataires des biens labellisés pour les conserver, les soutenir, produire des activités pour en augmenter la visibilité et générer des retombées positives dans de nombreux domaines. D’autre part, le prix du paysage de la CEP qui est attribué à une « réalisation effective et mesurable » d'activités et de comportements vertueux de sauvegarde et d'intégration du paysage dans l'optique de la Convention.

16 La promotion de la sensibilisation, de la formation, la reconnaissance du rôle fondamental de la connaissance du paysage, de l'analyse de ses transformations, l'insertion dans les enseignements scolaires et universitaires des «valeurs attachées au paysage » (CEP, art. $6 \mathrm{~B}, \S \mathrm{c}$ ) se taillent la part du lion dans cette Convention. Mais d'autres points méritent aussi notre attention.

17 Proche du paysage, le cadre de vie, c'est-à-dire « les conditions matérielles, sociales, économiques et culturelles » dans lequelles se trouvent les populations, actrices principales du paysage pour la CEP, comme nous l'avons vu. Ce cadre de vie influe sur le bien-être individuel et social, dont le paysage est un élément essentiel. Notions analogues, cadre de vie et paysage ne sont pas toujours perçus comme étant liés. La Recommandation CM/ Rec(2008)3 les mentionne pour rappeler que des perceptions différentes les sous-tendent. Le cadre de vie concerne davantage un ressenti individuel des données paysagères comme bien-être, alors que le paysage, en tant que «partie de territoire telle que perçue par les populations », est rapporté au bien-être social.

\section{Le Glossaire}


18 Le Glossaire du Système d'information de la Convention européenne du paysage est une réponse à la nécessité de fournir une aide à tous ceux (autorités, organismes, citoyens) qui cherchent à définir et à réaliser les données de la CEP par des politiques appropriées, à saisir la diversité des outils et des expériences utiles pour mieux connaître les paysages et les définir dans leurs spécificités. Rédigé en français, il est organisé par groupes d'items lexicaux (par exemple : " Paysage », "Structures paysagères / structure du paysage », "Éléments de paysage », "Zone urbaine », " Urbanisme », etc.) qui caractérisent une meilleure délimitation de la notion de paysage en le rapportant à son aménagement, au territoire, à l'écologie, items qui précisent les objectifs, processus et moyens par lesquels les Européens s'investissent dans des politiques participatives, et qui identifient des domaines spécifiques à la CEP en les comparant aux données d'autres traités internationaux.

Les versions 2012 et $2013^{11}$ que nous avons examinées montrent cette mise à plat, pour chacune des données lexicales prises en considération, avec les mêmes données et leurs occurrences dans les nombreux traités internationaux impliqués pour les comparaisons. L'annexe de la version 2013 liste les vingt-deux traités utilisés, outre, évidemment, la CEP et la Recommandation CM/Rec(2008) $3^{12}$.

Le tableau en page suivante sert d'exemple et concerne le mot vedette " patrimoine » dans la version du 15 février 2013 du Glossaire.

\begin{tabular}{|c|c|c|}
\hline \multirow{4}{*}{ Patrimoine } & $\begin{array}{l}\text { Définition } \\
\text { en langage } \\
\text { courant du } \\
\text { CEP }\end{array}$ & $\begin{array}{l}\text { "Ensemble des biens d'un groupe, d'une communauté, d'une } \\
\text { collectivité. Le patrimoine est indissociable de la notion de } \\
\text { transmission aux générations futures d'un héritage reçu des } \\
\text { générations passées. Dans ce sens, le paysage, qu'il soit } \\
\text { remarquable, du quotidien ou dégradé, en tant que bien } \\
\text { commun, est un patrimoine qui sera transmis aux générations } \\
\text { futures. Cette transmission ne concerne pas seulement l'héritage } \\
\text { du passé, elle comprend aussi les interventions de la génération } \\
\text { actuelle, pour le meilleur comme pour le pire. » }\end{array}$ \\
\hline & $\begin{array}{l}\text { Définition } \\
\text { Unesco }\end{array}$ & $\begin{array}{l}\text { Focalisation sur le patrimoine (matériel) mondial : « Le } \\
\text { patrimoine, qu'il soit architectural, archéologique, naturel ou } \\
\text { culturel, est constitué de biens considérés comme } \\
\text { remarquables. » }\end{array}$ \\
\hline & $\begin{array}{l}\text { Consensus } \\
\text { d'autres } \\
\text { conventions } \\
\text { sur la } \\
\text { définition } \\
\text { Unesco }\end{array}$ & $\begin{array}{l}\text { - Convention pour la sauvegarde du patrimoine architectural de } \\
\text { l'Europe (Grenade, 1985). } \\
\text { - Convention pour la sauvegarde du patrimoine archéologique } \\
\text { (révisée) (La Valette, 1992). }\end{array}$ \\
\hline & $\begin{array}{l}\text { Variantes de } \\
\text { la définition } \\
\text { Unesco }\end{array}$ & $\begin{array}{l}\text { - Convention-cadre du Conseil de l'Europe sur la valeur du } \\
\text { patrimoine culturel pour la société (Faro, 2005) : «La définition } \\
\text { du patrimoine culturel [est] élargie au-delà de la notion de bien et } \\
\text { du critère esthétique ou remarquable. » } \\
\text { - Orientations pour la mise en œuvre de la Convention } \\
\text { européenne du paysage (2011) : « Le patrimoine culturel et } \\
\text { historique est “inséré ” dans le paysage, c'est-à-dire qu'il en est } \\
\text { l'une des composantes. La Convention européenne du paysage } \\
\text { en effet concerne tant les paysages pouvant être considérés } \\
\text { comme remarquables, que les paysages du quotidien et les } \\
\text { paysages dégradés. » } \\
\text { La CEP « considère le paysage comme une composante ou } \\
\text { l'expression d'un patrimoine plus global, que ce soit celui de } \\
\text { l'Europe ou celui des populations. » }\end{array}$ \\
\hline
\end{tabular}

Ces retouches, ajouts et commentaires ne peuvent qu'intéresser au plus haut point les linguistes et permettent d'analyser la part langagière des traités internationaux. Cette part est perceptible dans ce va-et-vient entre occurrences, et permet de voir que, comme pour toute pratique langagière, nous avons là une pratique sociale, qui « n'est pas réductible à une pure description ou explication du monde, mais [...] a une puissance d'action sur celui-ci ; non seulement le langage dit le monde, mais le langage transforme, modifie, façonne ce monde » (Boutet, $2010: 11$ ). 
22 De plus, considérant que la CEP est rédigée en anglais et en français, l'intitulé «Versions linguistiques » parmi les commentaires du Glossaire contient des différences sémantiques du fait des traductions : les traductions de la CEP et des concepts qu'elle comporte sont parfois affaiblies par des traductions littérales ou trop rapides. Les deux textes originaux de la CEP eux-mêmes ne sont pas des traductions littérales de l'un à l'autre. Ainsi, pour la définition même de paysage, au français "partie de territoire » correspond l'anglais area. Parfois, les écarts entre les versions linguistiques sont plus importants : le Protocole d'application de la convention alpine de 1991 dans le domaine de la protection de la nature et de l'entretien des paysages a pour titre anglais Protocol Conservation of Nature and the Countryside ${ }^{13}$. De même, sont soumises à l'attention des lecteurs les traductions en anglais de " cadre de vie des populations » (surroundings), « intégration du paysage dans les politiques sectorielles » (to integrate landscape) (art. 5), " espace, milieu, territoire » (area) (seul mot utilisé en anglais comme alternative à landscape).

\section{Patrimoine et paysage vs paysage et patrimoine}

La notice du Glossaire ayant comme mot vedette " patrimoine », comme nous l'avons vu précédemment, a fait ressortir des glissements sémantiques dans l'interprétation de ce concept essentiel pour la CEP et pour tout traité international dédié à cette question, même par renvois. Au-delà de la définition du patrimoine en langage courant par laquelle débute la notice, et en amont de réflexions que nous allons avancer, le Dictionnaire culturel de la langue française (Rey, 2005 : t. III, s. v. patrimoine) détaille l'étymologie (du latin pater, les biens hérités du père), les acceptions du droit (fortune), de la biologie (caractères transmis par les gènes), l'acception métaphorique où apparaissent comme exemples «patrimoine de l'humanité » et " patrimoine mondial de l'humanité ", preuve, si besoin est, de la vitalité en lexicalisation de ces deux mots composés, redevable à leur large diffusion liée au succès de la Convention Unesco.

Le Glossaire met en relief les différents degrés de positionnement du patrimoine et du paysage : pour la CEP, qui privilégie la dimension européenne, sans empêcher une dimension mondiale, le paysage, dans ses aspects culturels, naturels, historiques - nous l'avons vu -, est une composante d'un patrimoine plus vaste, l'Europe, où les populations elles-mêmes (nous soulignons le rôle de protagoniste accordé à ces dernières) sont sur le devant de la scène. La Convention Unesco considère que le paysage, en raison de ses données culturelles, naturelles et historiques, est un patrimoine.

Cette Convention détaille donc, dans ses articles 1 et 2, les biens qui sont à considérer comme « patrimoine culturel » et comme «patrimoine naturel » ${ }^{14}$ : ces deux articles définissent un dénominateur commun d'exceptionnalité et de valeur universelle. Indéniablement, ces caractéristiques ont permis d'accélérer la sensibilisation à des biens matériels et immatériels hors du commun, méconnus parfois, auxquels l'entrée dans la liste du patrimoine Unesco de l'humanité a donné cadrage institutionnel, visibilité internationale, spectacularisation, ressources et retombées économiques. Responsabilité aussi, à l'égard de cette nomination d'exception, et à l'égard des générations à venir.

En ce qui concerne le patrimoine culturel, la Convention de Faro (2005) ${ }^{15}$ oriente ses articles sur la responsabilité et le respect communs (« de toute personne ») envers le patrimoine culturel des autres, de soi-même et « en conséquence, le patrimoine culturel de l'Europe » (art. 4). Ce patrimoine représente une « source partagée de mémoire, de compréhension, d'identité, de cohésion, de créativité » (art. 3).

Dans la Convention de Faro, l'élargissement de la définition de patrimoine culturel à l'environnement et à tous ses aspects, y compris les interrelations entre les personnes et l'évolution à travers le temps, conduit à affirmer que reconnaître le patrimoine culturel ouvre la voie à la construction pacifique et démocratique de la société, au développement durable, à l'accueil et à la promotion de la diversité culturelle. 
28 Ces parcours discursifs confirment les valeurs morales positives qui foisonnent dans les conventions et les traités internationaux : pour la Convention de Faro, nous avons relevé les locutions suivantes : " développement durable », " utilisation durable », " édification d’une société pacifique et démocratique ». Remarquons, dans ce cas, l'enrichissement du paradigme des compositions nominales nom + adjectif, dont le second élément est « durable ».

Cette Convention est proche des articles de la CEP et de ses objectifs. La fabrique du patrimoine européen repose sur tout un réseau d'actions de sensibilisation, de bonnes pratiques visant des biens matériels et immatériels pour lesquels la CEP pose des principes d'interprétation et d'agissements. Le principe démocratique est actualisé en accordant la première place aux populations européennes comme partie prenante dans l'élaboration de leur cadre de vie. Autre principe non négociable : le développement durable pour un environnement régulé et un développement économique soucieux de la reproduction des ressources, de « l'équité sociale et intergénérationnelle » (Luginbühl, A5, 2007 : 105).

30 L'immense potentiel patrimonial des paysages en Europe - qui, surtout en Europe, ne sont redevables ni à des facteurs uniquement naturels ni à des facteurs uniquement humains - nécessite une politique que la CEP déclare dans son Glossaire (version du 4 janvier 2013) ne pas être a priori centrée sur les interventions, et ne pas être assimilable à une politique sectorielle qui se cumulerait avec d'autres politiques sectorielles des pays européens, des institutions, des régions et autres autorités locales.

\section{Insaisissable paysage ?}

Dans la CEP, la politique du paysage, définie par l'article $1 \mathrm{~b}$ en tant que « protection, gestion et aménagement du paysage », est fondée sur des principes généraux, des stratégies et des orientations, modulés tous les trois par les objectifs de qualité paysagère. Si les objectifs de qualité paysagère sont peu définis et restent flous dans le texte instituant de la CEP, la Recommandation CM/Rec(2008)3 (I.1.H) laisse entendre que tout projet d'intervention sur le territoire devrait viser l'amélioration de la qualité paysagère ou, a minima, éviter de la réduire. Le cinquième atelier du Conseil de l'Europe pour la CEP (A5, 2007), dédié aux « objectifs de qualité paysagère » ${ }^{16}$, est un révélateur non seulement de multiples réflexions définitoires desdits objectifs, mais aussi des témoignages d'actualisation de projets dans différents pays européens, et même dans d'autres continents - l'Australie - en quête d'équilibre dans les choix politiques pour la sauvegarde de l'identité paysagère (Furze, A5, 2007).

Se pencher sur les objectifs de qualité paysagère renvoie au principe de la CEP qui se réfère aux paysages - cadre de vie des populations qui les habitent. Étroitement liée à ce principe, on trouve l'acceptation des diverses « perceptions » (individuelles, communautaires, sociales) que les populations peuvent avoir à l'égard du territoire où elles vivent (Luginbühl, A5, 2007). Ces populations seraient, selon ce texte, à égalité dans le droit d'exprimer leur ressenti, sans priorités pour qui détiendrait plus de pouvoir.

33 En reprenant de façon ponctuelle ces objectifs de qualité paysagère, une des analyses de cet atelier expose des processus indispensables pour leur mise en ouvre :

- $\quad$ processus d'identification : recherche et définition des caractères essentiels d'un paysage dans son état actuel et dans son développement futur. D’où, par exemple, la promotion des atlas et des cartographies ;

- $\quad$ processus de qualification, relatif à la valeur que les populations accordent au paysage. Les groupes sociaux ayant reconnu les caractères identifiants d'un paysage sont censés reconnaître et respecter l'hétérogénéité des perceptions dudit paysage. Mettre en discours ces perceptions est une difficulté majeure, révélée linguistiquement par des items qui tournent en boucle, tels que :

- pour « Objectif de qualité paysagère » : « formulation [...] des aspirations des populations en ce qui concerne les caractéristiques paysagères de leur cadre de vie »; 
- pour «Formulation d'objectifs de qualité paysagère » : « il s'agit de formuler des objectifs de qualité paysagère pour les paysages identifiés et qualifiés, après consultation du public ».

34 Le paysage est aussi une configuration géographique, une forme dans le cadre d'un territoire, ce que rappellent les notices du Glossaire (version du 8 février 2013) ponctuant les différences entre « espace » et « territoire ». L'espace est défini en tant que «partie de la surface terrestre [...] une étendue biophysique » plus ou moins délimitée, alors que le territoire est à considérer d'après " la manière dont les populations se sont approprié un espace donné, par des systèmes juridiques et sociaux ». Ensuite, le paysage est à nouveau défini, avec une certaine redondance, comme partie du territoire sur laquelle les populations (et non seulement celles qui y vivent) portent leurs perceptions, leurs appréciations (c'est-à-dire les valeurs qu'elles lui reconnaissent) et en font l'objet de leurs aspirations, un de leurs droits identitaires les plus importants, le droit de bien-être, ou au moins de mieux-être. Sans oublier l'écologie et la biodiversité, qui font partie des thèmes majeurs de notre époque : la Convention des Nations unies sur la diversité biologique (1992) ${ }^{17}$ définit une écologie du paysage comme discipline. À la différence de la CEP, pour qui la perception du paysage de la part des populations est plus globalisante que les délimitations d'une discipline.

35 Réalité géographique et patrimoniale, mais aussi sans conteste richesse culturelle, le paysage est un enjeu symbolique déterminant l'identité de tous les pays et de l'Europe tout entière. Associer paysage (dimension esthétique comprise) et identité conduit la CEP à utiliser un certain nombre d'expressions imagées, qui renvoient au visage comme critère majeur d’identification (cf. Martínez de Pisón, A4, 2006 : 278).

\section{Conclusion}

36 La CEP a fait l'objet de critiques, entre autres l'accusation de flou de certains items lexicaux et phrastiques du texte instituant, avec un spectre sémantique trop large et par conséquent imprécis. Des contradictions aussi s'y trouveraient :

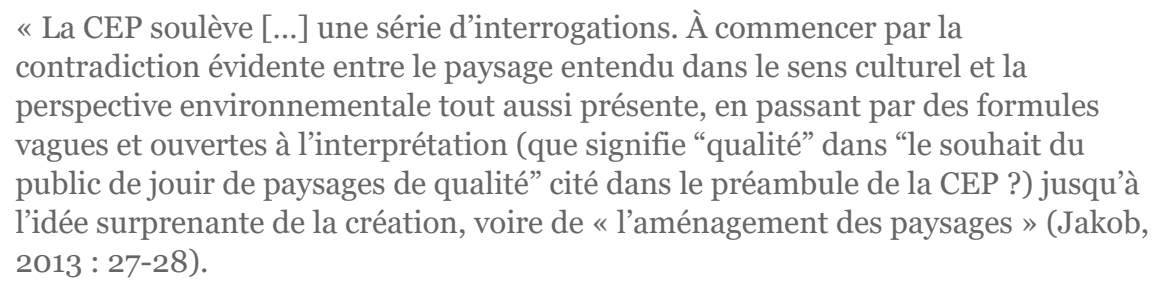

Difficulté aussi (cf. art. 2 de la CEP) concernant les " espaces naturels », cités sans plus de précision sur le sens et le référencement de l'adjectif « naturels ». Un certain flou, donc, peut-être pour éviter des dissensions notionnelles et politiques.

Ce flou est une des caractéristiques du lissage de la rédaction finale des textes et discours institutionnels, comme nous l'avons signalé en ouverture d'article. Il produit une "langue de coton ", selon une belle formule très utilisée dans la littérature scientifique. Le recours au « vague » de notions générales montre en filigrane qu’il est le résultat d'une médiation constante, construite à petits pas entre les membres de l'institution qui signent la convention ou le traité. Rappelons-le, la CEP est un document de politique publique du paysage européen qui amène à la reconnaissance juridique du paysage « cadre de vie des populations » (cf. Périgord \& Donadieu, 2012 : 47).

39 Même les textes du droit connaissent ce flou (cf. Bhatia et al., 2005), qui engendre interprétations et contradictions. Le texte instituant de la CEP a un empan très large sur l'Europe et sur ses États membres, ses articles poursuivent une vue commune, européenne, mais respectueuses des institutions locales, régionales et nationales. Entreprise ardue qui essaie d'embrasser tous les possibles, parfois les contraires, et des divergences importantes. 
40 Le passage sur les politiques du paysage prévoit d'intégrer la dimension paysagère dans toutes les politiques sectorielles qui ont trait à la transformation du territoire, de soutenir et d'articuler la collaboration - surtout dans de nouvelles formes - « entre les organismes et les différents niveaux de l'administration »(CEP, I.4). Sur le plan opérationnel, même le doigté politique le plus expert devrait prévoir des compromis. L'affirmation de l'identité locale pourrait aboutir à des fermetures avec exclusion d'identités plus larges.

41 À les lire au pied de la lettre, bien des textes des conventions internationales semblent utopiques, c'est-à-dire " "faire signe" vers un monde possible, souhaitable et vraisemblable» (Danblon, $2013:$ 214-215). Néanmoins, leur flou linguistique est non seulement une stratégie communicationnelle, mais aussi une richesse, par sa flexibilité, par les marges qui sont accordées à des interprétations non réductives, soucieuses de bien agir.

42 Dans un bel article, Bruno Vecchio (2011) se penche sur les procédures de participation du public énoncées par la CEP et sur l'enjeu social qui en découle. Il analyse le focus sur l'aspect identitaire du paysage où convergent la subjectivité et la socialité des habitants, surtout lorsqu'on veut donner sens et quelque précision à " perception », mot-clé pour déterminer la valeur que les populations attribuent à leur cadre de vie, aux paysages, urbains ou non urbains, quotidiens, dégradés ou de qualité. Des enquêtes de terrain, relate Vecchio, ont été menées sur le « vécu du paysage » par les populations, qui ont révélé les difficultés d'appréhension et d'expression de la perception des paysages par les individus. Sensibilité, émotions, flux d'impressions ont du mal à atteindre le seuil langagier : souvent " les mots manquent », les personnes enquêtées ne parviennent pas à exposer leurs perceptions.

Cet enjeu des perceptions individuelles et sociales des populations est difficile à atteindre : tout un réseau de savoirs (anthropologie, sémiotique du paysage...) pourrait être mis à contribution pour un aménagement où l'interprétation structurelle, formelle, rencontrerait et partagerait le ressenti existentiel des populations par qui le paysage acquiert sa définition.

\section{Bibliographie}

Bhatia (Vijay K.), Engberg (Jan), Gotti (Maurizio) \& Heller (Dorothee). 2005. Vagueness in Normative Texts. Berne : Peter Lang.

Boutet (Josiane). 2010. Le Pouvoir des mots. Paris : La Dispute.

Danblon (Emmanuelle). 2013. L'Homme rhétorique : Culture, raison, action. Paris, Le Cerf.

Furze (Brian). 2007. "Landscape values in Australia », p. 349-354 in Cinquième réunion des Ateliers du Conseil de l'Europe pour la mise en œuvre de la Convention européenne du paysage (Girone, 28-29 septembre 2006). Strasbourg : Council of Europe Publishing.

Jakob (Michael). 2013. Le Paysage. Gollion (Suisse) : Infolio Éditions.

Krieg-Planque (Alice). 2012. Analyser les discours institutionnels. Paris : Armand Colin.

Luginbühl (Yves). 2007. «Les objectifs de qualité paysagère », p. 105-113 in Cinquième réunion des Ateliers du Conseil de l'Europe pour la mise en œuvre de la Convention européenne du paysage (Girone, 28-29 septembre 2006). Strasbourg : Council of Europe Publishing.

Margarito (Mariagrazia). 2011. " “... comme un beau paysage” : de la Convention européenne du paysage et de quelques nouvelles axiologies ", p. 281-291 in La Comunicazione turistica. Lingue, culture, istituzioni a confronto. La Communication touristique. Langues, cultures, institutions en face-à-face / sous la direction de Mariagrazia Margarito, Marie Hédiard \& Nadine Celotti. Turin : Edizioni Libreria Cortina.

Martínez de Pisón (Eduardo). 2006. « La signification culturelle du paysage », p. 277-283 in Cinquième réunion des Ateliers du Conseil de l'Europe pour la mise en ouvre de la Convention européenne du paysage (Girone, 28-29 septembre 2006). Strasbourg : Council of Europe Publishing.

Oger (Claire) \& Ollivier-Yanniv (Caroline). 2003. « Du discours de l'institution aux discours institutionnels : vers la constitution de corpus hétérogènes », Xe Colloque bilatéral francoroumain, CIFSIC Université de Bucarest, 28 juin au 3 juillet 2003. En ligne : https://archivesic.ccsd.cnrs.fr/sic_ooooo717/document [consulté le 24 octobre 2019].

Oger (Claire) \& Ollivier-Yanniv (Caroline). 2006. « Conjurer le désordre discursif. Les procédés de "lissage" dans la fabrication du discours institutionnel ». Mots. Les langages du politique, 81, 
p. 63-67. En ligne : https://archivesic.ccsd.cnrs.fr/sic_ooooo717/document [consulté le 24 octobre 2019].

Périgord (Michel) \& Donadieu (Pierre). 2012. Le Paysage. Entre natures et cultures. Paris : Armand Colin.

Rey (Alain) (dir.). 2005. Dictionnaire culturel en langue française. 4 t. Paris : Le Robert.

Spingola (Felice). 2007. « Paysage et changement : vers un nouveau modèle de gouvernance dans les politiques du paysage », p. 237-246 in Quatrième réunion des Ateliers du Conseil de l'Europe pour la mise en œuvre de la Convention européenne du paysage (Ljubljana, 11-12 mai 2006). Strasbourg : Council of Europe Publishing.

Vecchio (Bruno). 2011. "Convenzione europea del paesaggio e progettazione paesaggistica. Alcuni problemi aperti », p. 305-325 in Lo Sguardo offeso. Il paesaggio in Italia storia geografia arte letteratura / sous la direction de Giovanni Tesio \& Giulia Pennaroli. Turin : Centro Studi Piemontesi.

\section{Sitographie}

(Les sites indiqués ont tous été consulté le 24 octobre 2019.)

Convention européenne du paysage : https://rm.coe.int/168008062a

Recommandation CM/Rec(2008)3 du Comité des ministres du Conseil de l'Europe aux États membres sur les orientations pour la mise en œuvre de la Convention européenne du paysage : https://rm.coe.int/CoERMPublicCommonSearchServices/DisplayDCTMContent? documentId=09000016802f8oca

Glossaire du Système d'information de la Convention européenne du paysage : https://rm.coe.int/16802fc145

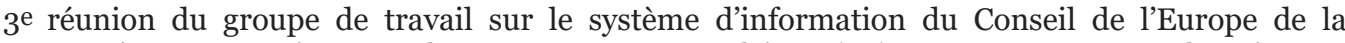
Convention européenne du paysage - Elcis (L6) - et son glossaire : https://rm.coe.int/CoERMPublicCommonSearchServices/DisplayDCTMContent? documentId=09000016802fdo24

Convention des Nations unies concernant la protection du patrimoine mondial culturel et naturel : https://whc.unesco.org/archive/convention-fr.pdf

Orientations devant guider la mise en œuvre de la Convention du patrimoine mondial : http://whc.unesco.org/fr/orientations/

Convention des Nations unies sur la diversité biologique : https://www.cbd.int/doc/legal/cbdfr.pdf

Conseil général de l'environnement et du développement durable : https://www.cgedd.developpement-durable.gouv.fr

\section{Notes}

1 I. Dispositions générales, II. Mesures nationales, III. Coopération européenne, IV. Clauses finales (https://rm.coe.int/168008062a).

2 Voir, ci-après, « Le glossaire ».

3 Les versions officielles sont en anglais et en français.

4 https://rm.coe.int/16802f8oc9.

5 Système d'information du Conseil de l'Europe sur la Convention européenne du paysage (ELC L6) (https://rm.coe.int/CoERMPublicCommonSearchServices/DisplayDCTMContent? documentId=09000016802fdo24) ; Glossaire du Système d'information du Conseil de l'Europe sur la Convention européenne du paysage (https://rm.coe.int/16802fc145).

6 Nous citons dorénavant les textes provenant de ces « Actes annuels des réunions » avec le sigle A (atelier) suivi d'un chiffre et de l'année. La bibliographie fournira les détails.

7 Quelques exemples de thèmes d'ateliers et de leurs Actes : « Les territoires du futur : identification et qualification des paysages, un exercice de démocratie » (2013) ; " Paysages et coopération transfrontalière : le paysage ne connaît pas de frontière » (2015) ; " Paysage et éducation » (2018); « Eau, paysage et citoyenneté face aux changements mondiaux » (2019).

8 " Considérant que le but du Conseil de l'Europe est [...] soucieux de parvenir à un développement durable [...], Notant que le paysage participe de manière importante à l'intérêt général [...], Conscients que le paysage concourt à l'élaboration des cultures locales [...] ».

9 https://whc.unesco.org/archive/convention-fr.pdf.

10 http://whc.unesco.org/fr/orientations/. 
1112 juin, 29 août et 3 novembre 2012, 4 janvier et 8 février 2013.

12 La Convention pour la sauvegarde du patrimoine architectural de l'Europe (Grenade, 1985), le Rapport Brundtland "Notre avenir à tous" (1987), la Convention des Nations unies sur la diversité biologique (Rio de Janeiro, 1992), la Convention pour la sauvegarde du patrimoine archéologique (révisée) (La Valette, 1992), la Convention sur l'accès à l'information, la participation du public au processus décisionnel et à l'accès à la justice en matière d'environnement (Aarhus, 1998), la Convention-cadre du Conseil de l'Europe sur la valeur du patrimoine culturel pour la société (Faro, 2005), le Glossaire Cemat [Conférence des ministres responsables de l'aménagement du territoire] du Conseil de l'Europe des expressions clés utilisées dans les politiques de développement territorial (2006), les Orientations devant guider la mise en œuvre de la Convention Unesco concernant la protection du patrimoine mondial, culturel et naturel (2011).

13 Glossaire, version du 29 août 2012.

14 « Art. 1 [patrimoine culturel] : les monuments : œuvres architecturales, de sculpture ou de peinture monumentales, éléments ou structures de caractère archéologique, inscriptions, grottes et groupes d'éléments, qui ont une valeur universelle exceptionnelle du point de vue de l'histoire, de l'art ou de la science ; les ensembles : groupes de constructions isolées ou réunies, qui, en raison de leur unité, ou de leur intégration dans le paysage, ont une valeur universelle exceptionnelle du point de vue de l'histoire, de l'art ou de la science ; les sites : œuvres de l'homme ou œuvres conjuguées de l'homme et de la nature, ainsi que les zones y compris les sites archéologiques qui ont une valeur universelle exceptionnelle du point de vue historique, esthétique, ethnologique ou anthropologique. Art. 2 [patrimoine naturel] : " les monuments naturels constitués par des formations physiques et biologiques ou par des groupes de telles formations qui ont une valeur universelle exceptionnelle du point de vue esthétique ou scientifique ; les formations géologiques et physiographiques et les zones strictement délimitées constituant l'habitat d'espèces animale et végétale menacées, qui ont une valeur universelle exceptionnelle du point de vue de la science ou de la conservation ; les sites naturels ou les zones naturelles strictement délimitées qui ont une valeur universelle exceptionnelle du point de vue de la science, de la conservation ou de la beauté naturelle. »

15 Convention du Conseil de l'Europe sur la valeur du patrimoine culturel pour la société.

16 Il apparait de façon manifeste combien ces ateliers sont un levain pour les travaux de la mise en œuvre du Glossaire.

17 https://www.cbd.int/doc/legal/cbd-fr.pdf

\section{Pour citer cet article}

Référence papier

Mariagrazia Margarito, « Dire le paysage-patrimoine dans la Convention européenne du paysage », Culture \& Musées, 33 | 2019, 157-179.

Référence électronique

Mariagrazia Margarito, « Dire le paysage-patrimoine dans la Convention européenne du paysage », Culture \& Musées [En ligne], 33 | 2019, mis en ligne le 19 novembre 2019, consulté le 08 juin 2021. URL : http://journals.openedition.org/culturemusees/3210 ; DOI : https://doi.org/10.4000/culturemusees.3210

\section{Auteur}

\section{Mariagrazia Margarito}

Université de Turin

Professeure émérite de linguistique française de I'Université de Turin (Italie). Ses domaines de recherche sont la lexicologie et la lexicographie françaises, notamment les italianismes du français, les études sur les stéréotypes. En analyse du discours, elle s'est occupée de textes touristiques, de textes expographiques et de discours des grandes institutions internationales. Parmi ses dernières publications : en 2013, «“... comme un beau paysage” : de la Convention européenne du paysage et de quelques nouvelles axiologies », p. 281-291 in La Comunicazione turistica. Lingue, culture, istituzioni a confronto. La Communication touristique. Langues, cultures, institutions en face-à-face / sous la direction de Mariagrazia Margarito, Marie Hédiard \& Nadine Celotti. Turin : Edizioni Libreria Cortina ; en 2013, « Marcher, courir : écriture et nonévénement », p. 285-294 in Dire l'événement. Langage mémoire société / sous la direction de Sophie Moirand, Sandrine Reboul-Touré, Danielle Londei \& Licia Reggiani. Paris : Presses Sorbonne nouvelle ; encore en 2013, « Dire l'émotion pour dire l'art », p. 41-56 in Comment parler de l'art? Approches discursives et sémiotiques / sous la direction de Mélissa BarkatDefradas \& Stéphanie Benoist. Paris : CNRS Éditions ; en 2014, «"Je suis dans le discours, donc j'existe....". L'objet d'art dans les textes d'accompagnement d'une exposition ». Labirinti, 154, "L'objet d'art et de culture à la lumière de ses médiations ", sous la direction de Jean-Paul Dufiet, p. 29-41. Tout récemment, en 2019 : « Exercices de vertus discursives. Textes 
expographiques à l'essai », p. 459-470 in Crisi della cultura e coscienza pedagogica / sous la direction de Elena Madrussan. Côme, Pavie : Ibis.

Courriel : mariagraziamargarito[at]gmail.com

\section{Droits d'auteur}

Culture \& Musées 